\title{
Gain a formal qualification and build your dental implant portfolio
}

With Ucer Education, you can train under the tuition of the most respected clinicians in the field of dental implantology for a Postgraduate Certificate in Implant Dentistry (EduQual Level 7) qualification.

Training also prepares you for the Diploma in Implant Dentistry of the Royal College of Surgeons of Edinburgh examination.

The comprehensive 12-month course covers core surgical and restorative dental principles of implant dentistry including the complete digital workflow, patient assessment and treatment planning.

Led by the highly esteemed and qualified education provider, Professor Ucer, the training is complemented by state-of-the-art facilities of a purpose built postgraduate
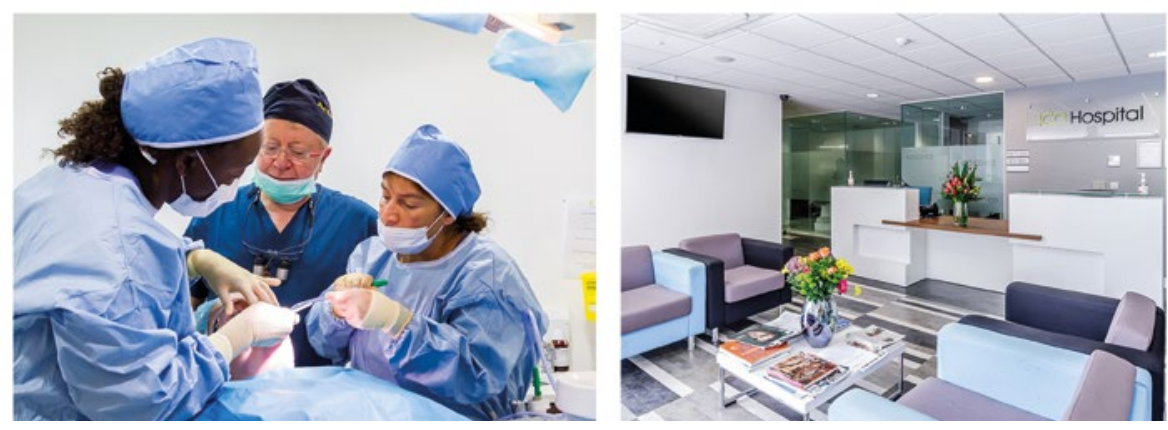

institute and on-going mentorship.

Delivered through the Manchester

Dental Implant Clinic at ICE Hospital and

Postgraduate Dental Institute in Salford

Quays, this is the ideal course for anyone

looking to develop their experience whilst obtaining a formal qualification in dental implantology.

More information is available by visiting www.ucer.education or calling 01612371842.

\section{Revolutionary intraoral scanning}

Dental professionals seeking a digital intraoral scanner that boasts enhanced accuracy, usability and speed should consider Dentsply Sirona's revolutionary new Primescan, available from trusted dental equipment supplier, Clark Dental.

Primescan sets new standards in digital intraoral scanning, offering the facility to export STL and exocad files to your preferred laboratory, or - in combination with the latest CEREC systems - to design restorations and produce sophisticated CEREC guides for dental implants.

When combined with CEREC milling units, Primescan is able to effectively support full chairside workflows for single-visit dentistry.

Featuring advanced smart pixel sensors and a state-of-the-art dynamic lens, Primescan is able to process more than one million $3 \mathrm{D}$ points per second, producing highly accurate, photorealistic images of the oral cavity.

With the ability to scan various dental materials - including amalgam, gold and glass - Primescan's increased field of view enables you to visualise larger areas with outstanding precision and minimal movement. High-resolution scans are instantly displayed on the touchscreen of Primescan's cutting-edge Acquisition Centre.

Primescan supports a wide range of dental procedures, from single-tooth to full-arch treatment plans.

More information is available by calling 01268733 146, emailing info@clarkdental. co.uk or visiting www.clarkdental.co.uk.

\section{Reduce chair time the easy way}

Do you find your glass ionomers are time consuming to prepare and use?

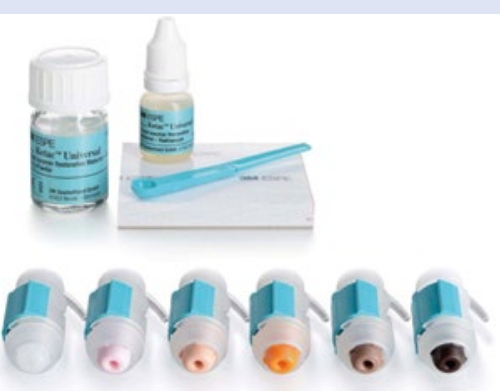

Make sure you keep it swift and simple with reduced chair time ${ }^{1}$ for your patients by choosing Ketac Universal glass ionomer restorative from 3M Oral Care.

Due to its innovative formula, Ketac Universal glass ionomer restorative requires no conditioner or coating. ${ }^{2}$

Furthermore, as the process requires less steps overall, ${ }^{3}$ practitioners can benefit from an easy to follow workflow and help patients, fast. It also makes an excellent alternative to amalgam.

For more information, call 0800626 578 or visit www.3M.co.uk/Dental.

$3 \mathrm{M}$ and Ketac are trademarks of the 3M Company.

\section{References}

1. 3M Oral Care Internal Data. Reduce chair time. Claim 6332. 2015.

2. $3 \mathrm{M}$ Oral Care Internal Data. No need for conditioner or coating. Claim 6331. 2015.

3. $3 \mathrm{M}$ Oral Care Internal Data. Few steps $=$ simple. Claim 6333. 2015. 